\title{
RATIONAL SPECTRAL FILTERS WITH OPTIMAL CONVERGENCE RATE
}

\author{
KONRAD KOLLNIG*, PAOLO BIENTINESI ${ }^{\dagger}$, AND EDOARDO DI NAPOLI ${ }^{\ddagger}$
}

\begin{abstract}
In recent years, contour-based eigensolvers have emerged as a standard approach for the solution of large and sparse eigenvalue problems. Building upon recent performance improvements through non-linear least square optimization of so-called rational filters, we introduce a systematic method to design these filters by minimizing the worst-case convergence ratio and eliminate the parametric dependence on weight functions. Further, we provide an efficient way to deal with the box-constraints which play a central role for the use of iterative linear solvers in contour-based eigensolvers. Indeed, these parameter-free filters consistently minimize the number of iterations and the number of FLOPs to reach convergence in the eigensolver. As a byproduct, our rational filters allow for a simple solution to load balancing when the solution of an interior eigenproblem is approached by the slicing of the sought after spectral interval.
\end{abstract}

Key words. Hermitian Eigenvalue Problem, Rational Filters, Contour-based Eigensolver, FEAST, Worst-case Convergence Rate, Load Balancing, Non-linear Least Squares, BFGS, NelderMead.

AMS subject classifications. 65F15, 41A20, 65Y05

1. Introduction. For the Hermitian eigenproblem $A x=\lambda x$ with $\lambda \in[a, b] \subset \mathbb{R}$, the last decade has seen the emergence of a new class of eigensolvers based on spectral projectors. Such eigensolvers are typically expressed as integrals of the spectral resolvent $(A-z I)^{-1}$ over a contour in the complex plane that encloses the interval $[a, b][32,33,31,9,21]$. Numerical quadrature transforms the contour integral into a matrix-valued rational function with complex coefficients $\beta_{i}$ and poles $z_{i}$. In this form, the problem of finding an efficient spectral projector is mapped to that of finding a rational function - often referred to as rational filter - that approximates the indicator function

$$
\mathbb{1}_{(a, b)}(x)= \begin{cases}1, & \text { if } x \in[a, b], \\ 0, & \text { otherwise. }\end{cases}
$$

This is a discontinuous function, often termed the "ideal filter", because it exactly maps the desired eigenvalues in the interval to 1 and the rest of the spectrum to 0 .

The algorithmic structure of eigensolvers based on rational filters has the advantage of lending itself to parallel implementations with multiple levels of nested parallelism $[8$, 6]. On the other hand, several factors make load balancing for these parallel eigensolvers a potential nightmare [10, 12]. Among them the design of the filter is an important element that influences the convergence of the eigensolver with direct consequences on the load balancing of any parallel implementation based on slicing $[a, b]$ in subintervals. In this paper, we focus on the design of filters with the aim of resolving this open issue. We build upon the results presented in [36] and introduce an optimization framework that is versatile and fast, eliminates parameter dependencies, and ultimately produces highly accurate rational filters with respect to a metric tightly bound to the quality of the ideal filter. Numerical tests show that an eigensolver equipped with our spectral projector converges with a rate that is practically independent from the search space size, the number of poles of the rational filter and the number of iterations required.

*RWTH Aachen University (konrad.kollnig@rwth-aachen.de).

†Umeå University (pauldj@cs.umu.se).

¥Jülich Supercomputing Centre, Forschungszentrum Jülich (e.di.napoli@fz-juelich.de). 
When $A$ is a Hermitian matrix, the corresponding rational filter is real-valued and symmetric with respect to the mapping $\left(x-x_{0}\right) \leftrightarrow\left(x_{0}-x\right)$, where $x_{0}$ is the center point of the interval $[a, b]$. Taking into consideration the complex conjugation and parity symmetries, Winkelmann et al. write $r$ as a rational function of order $(4 m-1,4 m)$,

$$
r(x):=r_{\beta, z}(x):=\sum_{i=1}^{m} \frac{\beta_{i}}{x-z_{i}}+\frac{\overline{\beta_{i}}}{x-\overline{z_{i}}}-\frac{\beta_{i}}{x+z_{i}}-\frac{\overline{\beta_{i}}}{x+\overline{z_{i}}}, \quad x \in \mathbb{R},
$$

where $m \in \mathbb{N}, \beta=\left(\beta_{1}, \ldots, \beta_{m}\right) \in \mathbb{C}^{m}$, and $z=\left(z_{1}, \ldots, z_{m}\right) \in\left(\mathbb{H}^{+R}\right)^{m}$, with $\mathbb{H}^{+R}$ being the right quadrant of the upper half of $(\mathbb{C} \backslash \mathbb{R})$ with origin in $x_{0}$. With this setup, the problem to be addressed is how to select, for a fixed degree $m^{1}$, the coefficients $\beta_{i}$ and the poles $z_{i}$ such that the corresponding rational function $r(x)$ approximates the ideal filter $\mathbb{1}_{(a, b)}$ according to a predetermined metric. Our aim is to build an optimization framework and select an appropriate metric such that the outcome is a filter $r$ stabilizing the convergence of the eigensolver.

Due to the discontinuity of the indicator function $\mathbb{1}_{(a, b)}$, the problem of determining the best coefficients and poles for $r(x)$ is tackled using a non-linear weighted leastsquares approach. For a given interval $[a, b]$, one aims to minimize the objective function

$$
f_{\omega}(\beta, z):=\int_{-\infty}^{\infty} \omega(x)\left(\mathbb{1}_{(a, b)}(x)-r_{\beta, z}(x)\right)^{2} \mathrm{~d} x, \quad \text { where } \beta \in \mathbb{C}^{m}, z \in\left(\mathbb{H}^{+R}\right)^{m}
$$

over $\beta$ and $z$ for some fixed $m \in \mathbb{N}$ and a weight function $\omega(x): \mathbb{R} \rightarrow[0, \infty)$, which is even and piecewise constant. This optimization framework, termed SLiSe in [36], provides a comprehensive parameterization of rational filters. The resulting SLiSe filters have proven to be competitive with previous rational filters, such as GaUssLEGENDRE [31] and ZOLOTAREV [12].

The SLiSe framework is independent of the specific eigensolver in which the function $r$ is plugged in and used as a spectral filter. At glance, a filter optimized through this framework should perform well independently from the target eigenproblem. In practice, the effectiveness of a filter depends indirectly from the eigenvalue distribution around the interval $[a, b]$ through the choice of the weight function $\omega$. In other words, despite its versatility, the SLiSe framework outputs filters whose quality is sensitive to the ad-hoc choice of weight functions and the piece-wise intervals defining them: small changes in the choice of $\omega(x)$ greatly influence the effectiveness of the resulting filter.

Contributions. Building on top of the SLiSe framework, this work addresses problematic aspects of such optimization and ultimately provides a solution to the open issue of how a spectral filters influence load balancing. In detail, we identify a number of main contributions. We improve the performance of the unconstrained minimization process by substituting the LEVENBERG-MARQUARDT with the BROYDEN-FLETCHERGoldFarb-Shanno (BFGS) algorithm [30, Chapter 6]. Likewise, when SLiSe is used in combination with box-constraints, it comes natural to extend BFGS to the L-BFGS-B algorithm [3, 38, 24]. Using the BFGS family of algorithms results in a substantial reduction of time-to-solution, which in turn is a necessary requirement to reduce the objective function residual and, at the same time, increases the accuracy of

\footnotetext{
${ }^{1}$ Strictly speaking the degree of $r$ is $4 m$. In the rest of the paper we will stick to a more intuitive notion of degree which refers to the number of poles in $\mathbb{H}^{+R}$ corresponding to the range of the index $i$ in Eq. (1.2)
} 
the SLiSe filters. We increase the accuracy by casting the problem of selecting $\beta_{i}$ s and $z_{i}$ S in terms of finding the corresponding rational function $r(x)$ that minimizes the Worse-case Convergence Rate (WCR) ${ }^{2}$. The relevance of this metric resides in the fact that the ideal filter $\mathbb{1}_{(a, b)}$ has the lowest possible value for $\mathrm{WCR}$, which is 0 .

In order to use the WCR metric effectively, we embed the SLiSe framework, equipped with the BFGS algorithm, within a second minimization process. This process has the explicit goal of minimizing the WCR metric with respect to the weight function $\omega$. We attain this target by using the derivative-free NELDER-MEAD algorithm. The by-product of this process is eliminating the dependence on the arbitrary choice of $\omega$ in the definition of the objective function $f_{\omega}(\beta, z)$. The net result is a parameter-free minimization framework with an enhanced usability and productivity. When used in interior eigensolvers based on subspace iteration, we observe that the rational filters obtained with the new minimization framework outperform state-of-the-art filters. The convergence rate of the eigensolver becomes almost independent from the size of the search subspace and the number of poles used. Consequently, the eigensolver is more robust in terms of convergence rate and does not require tweaking of the parameters associated with the spectral projection. In turn, this enhanced behavior of the eigensolver facilitates the load balancing when executed on parallel platforms. We termed this enhanced minimization framework, and the corresponding rational filters it produces, WiSe.

Related work. The interpretation of spectral projectors as rational (filter) functions of matrices was discussed in [35] for FEAST, and in [14, 13] for Sakurai-Sugiura-type eigensolvers. Rational filters were also proposed early on for signal processing by Murakami [29, 28, 25, 27, 26]. In recent years, filters have been treated as a parameter that can be designed via optimization methods. Van Barel [1] suggested a non-linear Least-Squares approach for non-Hermitian filters to be used in the Sakurai-Sugiura framework, while Xi and Saad [37] described linear Least-Squares optimized filters for the Hermitian FEAST eigensolver. Van Barel's approach is based on the discrete $\ell_{2}$ norm, not a functional approximation approach, and does not support constraints optimization. Xi and Saad present a linear Least-Squares minimization method where only the coefficients of the rational function are optimized. For FEAST, Güttel et al. presented a first approach to minimizing the WCR in Eq. (2.7). They derived a set of generalized GAUSS-LEGENDRE filters, parameterized by one variable only, with respect to which they minimized the WCR functional. The resulting WCR values were smaller than for unparameterized GAUSS-LEGENDRE filters, but not as small as for Zolotarev filters [31, 12, 2, 11] which offered the best WCR so far. This observation motivated a more rigorous parameterization of a subset of rational filters, that is, SLiSe filters [36], so as to benefit from a reduced number of parameters within WCR minimization.

Organization. The remainder of this paper is organized as follows. In Sec. 2 we introduce the reader to spectral filters and the general mathematical setup. In Sec. 3, we review the SLiSe framework and introduce efficient imposition of box-constraints on rational filters through L-BFGS-B. In Sec. 4, we illustrate the minimization scheme to reduce the WCR of SLiSe filters, which in turns eliminates the dependence on weight functions. In Sec. 5, we present a set of numerical experiments comparing our new filters to the state-of-the-art and illustrate their numerical properties and advantages. The last section summarizes our results and provides a perspective on their impact on the load balancing of parallel interior eigenvalue solvers.

\footnotetext{
${ }^{2}$ This metric is defined in the next section.
} 


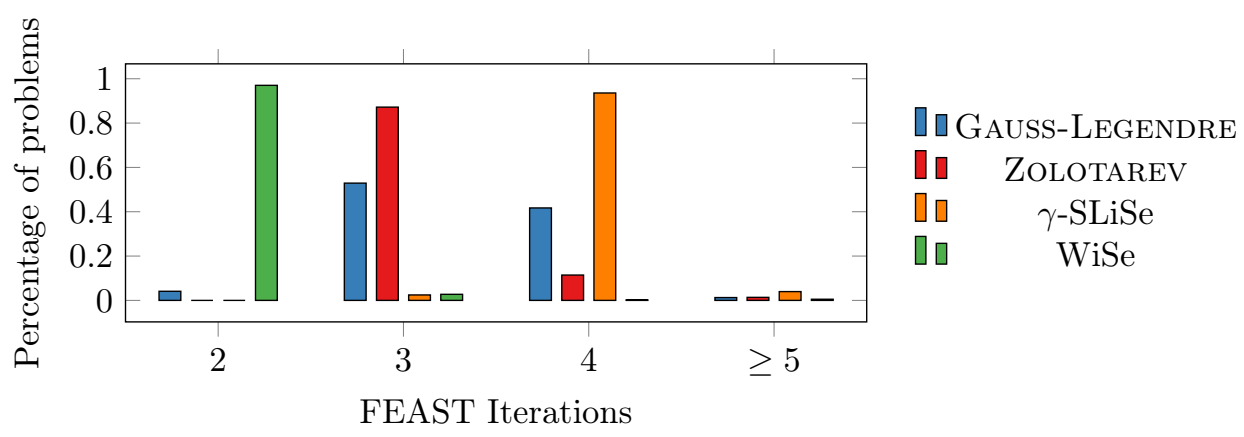

FIG. 1. FEAST iterations for different filters with gap parameter $G=0.95$ to solve 2117 benchmark eigenproblems, for an eigencount multplier of $C=1.1$. Our new WiSe filter outperforms the others, i.e. generalized Gauss-Legendre [31, 12], Zolotarev [12], and $\gamma$-SLiSe [36]. Details are discussed in Section 5.2.2.

2. Methodology. Contour-based eigensolvers were originally conceived for the solution of the generalized interior eigenvalue problem

$$
A v=\lambda B v, \quad \lambda \in[a, b],
$$

where $A, B \in \mathbb{C}^{n \times n}$ are Hermitian and $B$ is positive definite, $v \in \mathbb{C}^{n} \backslash\{0\}, a<b$ and $n \in \mathbb{N}$. A spectral projector can be defined as the integral of the matrix resolvent $(A-z B)^{-1}$ along a contour $\Gamma$ in the complex plane $\mathbb{C}$ enclosing the interval $[a, b] \subset \mathbb{R}$. Without loss of generality, one can linearly map $[a, b]$ to the standard interval $[-1,1]$ and select an integration contour around it. It is standard practice to compute the contour integral via numerical quadrature (e.g. GaUss-LEGENDRE)

$$
r(A, B):=\sum_{i} \beta_{i}\left(A-B z_{i}\right)^{-1} B \approx \frac{1}{2 \pi i} \oint_{\Gamma} \frac{\mathrm{d} z}{A-B z} B,
$$

with $\beta_{i}, z_{i} \in \mathbb{C}$. When used in combination with a subspace iteration scheme, $r(A, B)$ projects a given set of vectors $Y$ onto an invariant subspace of the spectrum corresponding to the eigenvalues within the interval $[-1,1]$ [18]. In practice, spectral projection exchanges the direct solution of the eigenproblem for that of many independent linear systems with multiple right-hand-sides

$$
\left(A-B z_{i}\right) V=\beta_{i} B Y .
$$

Because each linear system can be solved independently from the others, this class of eigensolvers naturally lends itself to multiple layers of parallelism, making contourbased eigensolvers especially well suited for today's increasingly parallel computer architectures. As shown in several recent publications, the performance of the eigensolver depends on the effectiveness of the spectral filter $r(A, B)[18,35,12,2,5,9]$. Recently, the authors of [36] proposed a numerical optimization approach alternative to the standard quadrature rules. By minimizing the objective function of Eq. (1.3), they propose a new class of rational filters, termed SLiSe, which perform better than the filters currently in use, on a large number of representative eigenproblems. Despite such an advance, the SLiSe framework showed a few shortcomings, such as slow convergence and lack of efficient support for box-constraints. These box-constraints - defined as upper and lower bounds on the imaginary parts of each $z_{i}$ - can substantially influence the time-to-solution in iterative linear system solvers. Having a time-to-solution 
comparable across all linear systems is a crucial element to load-balance a parallel eigensolver based on spectral projection.

The SLiSe filters. The SLiSe minimization framework aims to approximate the indicator function $\mathbb{1}_{(-1,1)}$ by rational filters $r(x)$ of a fixed degree $m$. This approximation is obtained by minimizing the objective function $f_{\omega}(\beta, z)$ from Eq. (1.3). In the SLiSe framework, a new filter is obtained as follows: Given a fixed weight function $\omega$ and an $m \in \mathbb{N}$, SLiSe takes an existing rational filter $r_{\bar{\beta}, \bar{z}}$, where $\bar{\beta} \in \mathbb{C}^{m}$ and $\bar{z} \in\left(\mathbb{H}^{+R}\right)^{m}$, and derives a new rational filter $r_{\hat{\beta}, \hat{z}}$, such that $(\hat{\beta}, \hat{z})$ solves the minimization problem

$$
\underset{\beta \in \mathbb{C}^{m}, z \in(\mathbb{H}+R)^{m}}{\operatorname{argmin}} f_{\omega}(\beta, z) .
$$

This minimization problem is non-linear and non-convex and therefore difficult to solve due to the non-existence of closed-form solutions. Yet, the objective function $f_{\omega}$, as well as its gradient

$$
\nabla f_{\omega}=\left(\nabla_{\beta_{1}} f_{\omega}, \ldots, \nabla_{\beta_{m}} f_{\omega}, \nabla_{z_{1}} f_{\omega}, \ldots, \nabla_{z_{m}} f_{\omega}\right)^{\top},
$$

are differentiable and can be computed through a small number of matrix operations [36]. In this setup, one can make use of a wide range of existing numerical minimization methods. Winkelmann et al. obtain SLiSe filters by employing two such minimization methods, gradient descent and LEVEnBERG-MARQUARDT (LM).

While LM makes for an effective minimization scheme, it may require up to thousands of iterations to converge to a satisfactory value for the residual level of $f_{\omega}$. Executing an efficient minimization becomes a pressing problem in the case of boxconstrained optimization, when the LM algorithm cannot be used and gradient descent requires up to millions of iterations to converge, which translates in a significantly larger amount of computing time over the unconstrained case. In addition, and most importantly, the quality of a resulting filter depends on the choice of weight function $\omega$, which is not automatic and requires an experienced user to follow a set of guidelines. In the following, we illustrate a minimization scheme that ensures speed of convergence, supports box-constraints, and eliminates the dependence on the custom choice of weight functions $\omega$.

The new minimization scheme. In the rest of the paper, we refer to $r$ as a rational filter, and, without loss of generality, consider only the case $r(A, B=I)=r(A)$. As seen in the previous section, if $A$ is a Hermitian matrix, the corresponding rational function $r(x)$ is forced to be real and symmetric and can be expressed with a subset of poles and coefficients as in Eq. (1.2). Since the minimization of the objective function in Eq. (1.3) is completely general, the resulting filter is independent of the specific subspace iteration eigensolver and can be plugged in any eigensolver of this type. Nonetheless, for practical purposes, we use the FEAST eigensolver [6] as a reference algorithm. Given and exact value $\lambda_{j} \in[-1,1]$, FEAST computes an approximate eigenpair $\left(\boldsymbol{q}_{j}, \hat{\lambda}_{j}\right)$ with a residual vector norm equal to $\left\|A \boldsymbol{q}_{j}-\hat{\lambda}_{j} \boldsymbol{q}_{j}\right\|$. Such residual converges linearly with a convergence rate given by $\left|\gamma_{\text {out }} / \gamma_{\text {in }}\right|$, where $\gamma_{\text {out }}\left(\gamma_{\text {in }}\right)$ is related to the maximum (minimum) value of the filter outside (inside) a neighborhood enclosing the $[-1,1]$ interval [35, Theorem 5.2]. Consequently, the convergence rate depends both on the spectrum of the given matrix $A$ and the spectral filter of choice.

Although the actual convergence rate will vary for different spectra, a filterdependent upper bound is given by the Worst-case Convergence Rate (WCR). The WCR applies to a variety of other eigensolvers based on spectral projection such as 
the block Sakurai-Sugiura-Rayleigh-Ritz method [33] and its non-iterative variant [15]. As defined in [12], the worst-case convergence rate satisfies the following theorem

TheOREm 2.1 ([12, Th.2.2]). Given a rational filter $r$ and a fixed gap parameter $G \in(0,1)$, the FEAST method converges linearly, with probability one, at a convergence rate no larger than

$$
w_{G}(r)=\frac{\max _{x \in\left[-\infty,-G^{-1}\right] \cup\left[G^{-1}, \infty\right]}|r(x)|}{\min _{x \in[-G, G]}|r(x)|}
$$

as long as no eigenvalues lie within $\left[-G^{-1},-G\right] \cup\left[G, G^{-1}\right]$. The occurring probability stems from choosing the initial subspace within the FEAST method at random.

Since Theorem 2.1 implies that $\left|\gamma_{\text {out }} / \gamma_{\text {in }}\right| \leq w_{G}(r)$, for an appropriate $G$, a smaller WCR value $w_{G}(r)$ implies faster worst-case convergence. As we already mentioned in the introduction, minimizing WCR for the SLiSe filters points out to which filters best approximate the ideal filter $\mathbb{1}_{(-1,1)}$ (that has indeed the optimal bound $w_{G}\left(\mathbb{1}_{(-1,1)}\right)=$ $0)$. Based on the considerations above, we can now define the following optimization problem.

Definition 2.2. Given $G \in(0,1), m \in \mathbb{N}$, and $r_{\beta, z}$ a rational filter as defined in Eq. (1.2), an optimal rational filter is one solving the minimization problem

$$
\underset{\beta \in \mathbb{C}^{m}, z \in(\mathbb{H}+R)^{m}}{\operatorname{argmin}} w_{G}\left(r_{\beta, z}\right) .
$$

In general, the WCR is a non-linear, derivative-free function. Its formulation makes it difficult to determine further mathematical properties, such as convexity or continuity. Conventional methods, like steepest descent, cannot be applied. Additionally, in derivative-free minimization, the number of $w_{G}$ function evaluations may become intractable very quickly, even for a modest increase of the rational filter degree $m$. These observations cause this minimization problem to be especially challenging. Instead of solving the problem as formulated in Eq. (2.7), we propose a modified minimization problem that combines the existing SLiSe framework, solving for $(\beta, z)$ while $\omega$ is fixed, as in Eq. (2.4), with minimization of the WCR with respect to $\omega$, seeking a better $\omega$ while $(\beta, z)$ is fixed. These two minimization problems

$$
\left\{\begin{array}{lll}
\beta, z & \leftarrow \underset{\beta, z}{\operatorname{argmin}} f_{\omega}(\beta, z) & \text { for a fixed } \omega \\
\omega & \leftarrow \underset{\omega}{\operatorname{argmin}} w_{G}\left(r_{\beta, z}(\omega)\right) & \text { for a fixed pair }(\beta, z)
\end{array}\right.
$$

are clearly not independent. The WCR it is minimized solely with respect to the weight function $\omega$-where we have indicated explicitly the dependence of the rational filter on the weight function in Eq. (2.8) - but it is a non-linear, derivative-free function. As such, the WCR depends on the whole $r$ which, in turn, depends on the minimization of the objective function $f_{\omega}$. In other words, we now have to solve two non-linearly dependent minimization problems, which need to be solved self-consistently. We will see in Section 4 how we implement this process in a nested loop fashion, where the SLiSe process is executed within the WCR minimization and convergence is reached self-consistently by continuously swapping between the two minimizations. Solving Eq. (2.8) is now a tractable problem, even if it calls for sophisticated algorithms, and is computationally very intensive, requiring many repeated invocations of the SLiSe minimization. 


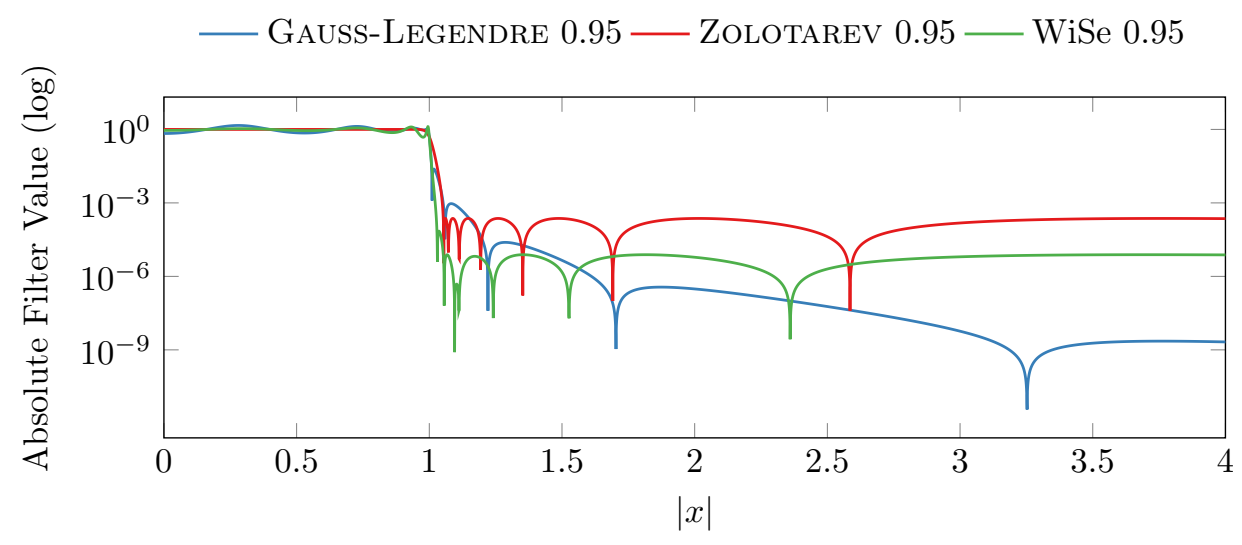

FIG. 2. Logarithmic plot of different 16-pole rational filters, showing the state-of-the-art GaussLEGENDRE and ZolotaReV rational filters [12, 31], alongside our new filter candidate, WiSe. A moderate gap parameter $G=0.95$ was chosen for Zolotarev, WiSe, and Gauss-Legendre. Our new WiSe filter provides the best WCR, which can be seen in offering the sharpest slump for $x$ around 1 , and maintaining a constantly close approximation of the indicator function $\mathbb{1}_{(-1,1)}$ across the whole domain of real numbers.

In order to increase the performance of the self-consistent minimization, we introduce the BFGS algorithm [30, Chapter 6] within the unconstrained SLiSe minimization process. Similarly, for box-constraints, we present an embedding of the L-BFGS-B minimization method $[3,38,24]$ into SLiSe. By formulating the WCR minimization as a nested process, we additionally solve the issue of weight function selection, which is one of the open issues of SLiSe. The net result is an extension of the SLiSe framework toward rational filters for faster convergence, without the need to select the weight functions by hand. Our new rational filters, termed WiSe, outperform state-of-the-art filters (see Figure 1 for experimental results and Figure 2 for filter plots). In particular, we prove that ZOLOTAREV filters do not provide best worst-case convergence, despite their optimality in approximating the indicator function with respect to the $\infty$-norm.

3. Efficient computation of WiSe filters. In this section, we introduce the use the BFGS algorithm, which yields faster convergence and better box-constrained rational filters than previous implementations. Moreover, the extended L-BFGS-B successfully addresses open issues that appear in box-constrained filters [12, 36, 9].

3.1. Accelerating SLiSe. When using the BFGS algorithm to solve the minimization problem in Eq. (2.4), we end up reducing substantially the number of function evaluations needed to reach convergence. Seemingly minor, this improvement is actually essential for an effective embedding of SLiSe into a scheme that is based on the minimization of the WCR. The BFGS algorithm belongs to the class of quasi-NEWTON methods. It approximates a local minimizer iteratively, in a manner similar to the popular GaUsS-NEWTON algorithm, which is Hessian-based. However, unlike Gauss-Newton, BFGS does not require the exact Hessian $\nabla^{2} f$, and uses an approximation instead. The minimum requirement for the algorithm to work is that the function $f$ has a quadratic expansion in Taylor series near the minimum. Thanks to this weaker condition, BFGS guarantees convergence also for non-smooth and non-convex functions.

The standard implementation of the BFGS-variant in Algorithm 3.1 does not 
offer support for real-valued objective functions of complex arguments, such as our $f_{\omega}$ from Eq. (1.3). This problem can be overcome by a conversion of $f_{\omega}$ and $\nabla f_{\omega}$ to real arguments. In the case of a generic function of a complex variable $g: \mathbb{C}^{n} \rightarrow \mathbb{R}$, one can separate the real from the imaginary parts [34] and instead minimize the function $\tilde{g}: \mathbb{R}^{2 n} \rightarrow \mathbb{R}$, defined as

$$
\tilde{g}\left(\left(\begin{array}{l}
a \\
b
\end{array}\right)\right):=g(a+i b), \quad \text { for } a, b \in \mathbb{R}^{n},
$$

by computing descent directions from its gradient

$$
\nabla \tilde{g}\left(\left(\begin{array}{l}
a \\
b
\end{array}\right)\right)=\left(\begin{array}{l}
\operatorname{Re} \nabla g(a+i b) \\
\operatorname{Im} \nabla g(a+i b)
\end{array}\right), \quad \text { for } a, b \in \mathbb{R}^{n} .
$$

The same mapping can be applied to the SLiSe functional $f_{\omega}: \mathbb{C}^{m} \times\left(\mathbb{H}^{+R}\right)^{m} \rightarrow \mathbb{R}$ because it operates on a subset of $\mathbb{C}^{2 m}$, where $m \in \mathbb{N}$ is the degree of the rational filter. In this case, one can think of the complex vectors $\beta$ and $z$ as being part of a vector $v=(\beta z)^{\top}$ and define $\tilde{f}: \mathbb{R}^{4 m} \rightarrow \mathbb{R}$ such that

$$
\tilde{f}\left(\begin{array}{l}
\left(\begin{array}{l}
\operatorname{Re}\left(\beta^{\top}\right) \\
\operatorname{Re}\left(z^{\top}\right)
\end{array}\right) \\
\left(\begin{array}{l}
\operatorname{Im}\left(\beta^{\top}\right) \\
\operatorname{Im}\left(z^{\top}\right)
\end{array}\right)
\end{array}\right):=f(\operatorname{Re}(\beta)+i \operatorname{Im}(\beta), \operatorname{Re}(z)+i \operatorname{Im}(z)) .
$$

Starting at an initial point $x_{0}=(\operatorname{Re}(\beta) \operatorname{Re}(z) \operatorname{Im}(\beta) \operatorname{Im}(z))^{\top}$, BFGS computes iterates $x_{k}$ that converge to a local minimizer of $\tilde{f}$ as $k \in \mathbb{N}$ increases, employing the descent directions

$$
p_{k}:=-H_{k} \nabla \tilde{f}\left(x_{k}\right),
$$

and a line search which guarantees that the secant condition is satisfied (see line 7 of Algorithm 3.1). $H_{k}$ is an approximation to the inverse Hessian of $\tilde{f}$ and is recursively defined as

$$
H_{0}:=I_{4 m}, \quad H_{k+1}:=\left(I_{4 m}-\frac{s_{k} y_{k}^{T}}{y_{k}^{T} s_{k}}\right) H_{k}\left(I_{4 m}-\frac{y_{k} s_{k}^{T}}{y_{k}^{T} s_{k}}\right)+\frac{s_{k} s_{k}^{T}}{y_{k}^{T} s_{k}}
$$

with

$$
s_{k}:=x_{k+1}-x_{k}, \quad y_{k}:=\nabla \tilde{f}\left(x_{k+1}\right)-\nabla \tilde{f}\left(x_{k}\right),
$$

where $x_{k}, s_{k}, y_{k}, p_{k} \in \mathbb{R}^{4 m}$ and $H_{k} \in \mathbb{R}^{4 m \times 4 m}$ for some $m \in \mathbb{N}$. The formulation through the BFGS algorithm converges faster than the previous minimization algorithms used by the SLiSe framework (see Figure 3 for the box-constrained case that is discussed in the following subsection). The conversion of the minimization functional to real-arguments allows one to use not only the BFGS scheme, but also various other minimization algorithms (such as those in the minimization algorithm collection NLOpt [16]). Despite such an advantage, most alternatives do not yield any substantial improvements over BFGS.

3.2. Imposing box-constraints efficiently. As described at the beginning of Section 2, the spectral projection at the base of the FEAST eigensolver leads to the solution of several independent linear systems with multiple RHS (see Eq. (2.3)). In 


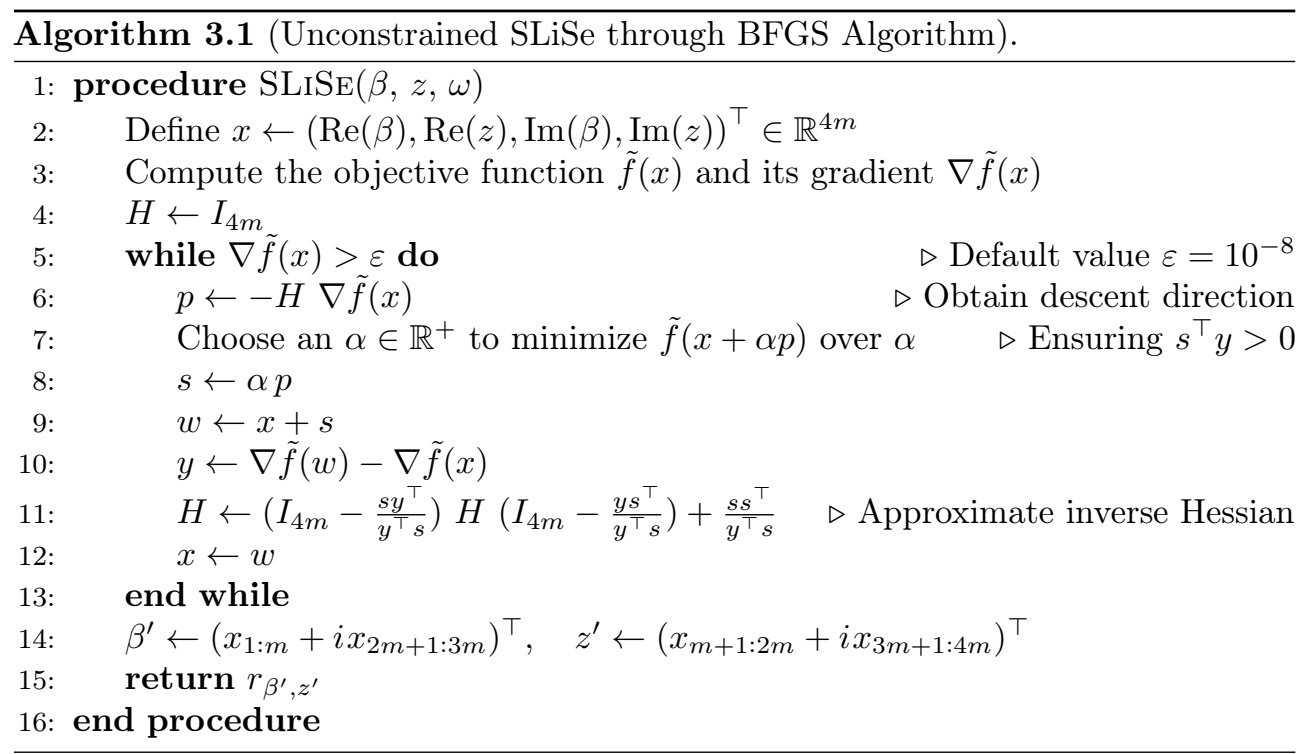

the case of very large and sparse systems, the use of direct solvers is not feasible due to memory requirements. In this case, iterative solvers, such as GMRES or CG, are the natural choice. For these methods, time-to-solution and accuracy depend substantially on the condition number of the resolvent matrices $\left(A-z_{i} I\right)$. When $A$ is Hermitian, such condition number is, up to a constant factor, equal to

$$
\kappa\left(A-z_{i} I\right)=\frac{\max _{\lambda_{a} \in \sigma(A)}\left|\lambda_{a}-z_{i}\right|}{\min _{\lambda_{b} \in \sigma(A)}\left|\lambda_{b}-z_{i}\right|} .
$$

Since the filter is built to approximate the indicator function $\mathbb{1}_{(-1,1)}$, the numerator of this equation is bound from above by $\left(\max _{\lambda_{a} \in \sigma(A)}\left|\lambda_{a}\right|+1\right)$, while the denominator is bound from below by $\left|\operatorname{Im}\left(z_{i}\right)\right|$. Consequently, if the poles of the rational function $r_{\beta, z}$ are close to the real axis, the condition number of some of the resolvent matrices can be quite high. This consideration motivated the introduction of the box-constraints $\left|\operatorname{Im}\left(z_{i}\right)\right| \geq \mathrm{lb}>0(i=1, \ldots, m)$ to the SLiSe minimization process, where $\mathrm{lb}$ is a positive constant representing the minimum distance of any pole from the real axis.

In the BFGS algorithm, box-constraints can be included by projecting the search direction onto the constraints. This is accomplished through the simple gradient projection $\mathcal{P}(x-t \nabla \tilde{f}(x))$, where $t>0$, followed by a BFGS update treating the bounded components of $x$ as equality constraints. In our case, the operator $\mathcal{P}: \mathbb{R}^{4 m} \rightarrow$ $\mathbb{R}^{4 m}$ projects only the imaginary part of the poles $\left|\operatorname{Im}\left(z_{i}\right)\right|$ and takes consequently the following form when acting on a vector $y \in \mathbb{R}^{4 m}$

$$
\mathcal{P}(y)_{j}:= \begin{cases}\operatorname{sign}\left(y_{j}\right) \cdot \mathrm{lb}, & \text { if }\left|y_{j}\right|>\mathrm{lb} \text { and } j \in\{3 m+1, \ldots, 4 m\}, \\ y_{j} & \text { otherwise }\end{cases}
$$

for $j=1, \ldots, 4 m$.

This approach is encoded in the L-BFGS-B algorithm, which extends projected gradient descent to the Hessian approximations from BFGS, and can be used to realize box-constrained minimization efficiently in SLiSe, similarly to what is done in Algorithm 3.1. The L-BFGS-B algorithm has shown to converge quickly in our 


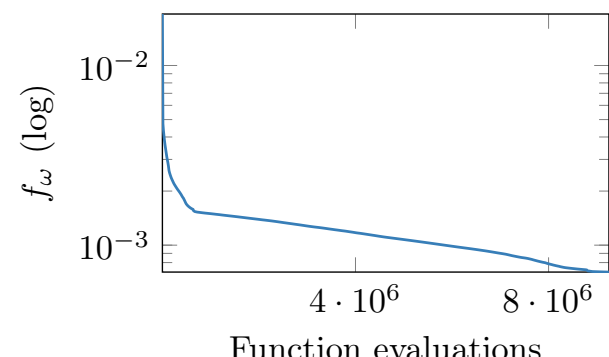

(a) Projected gradient descent

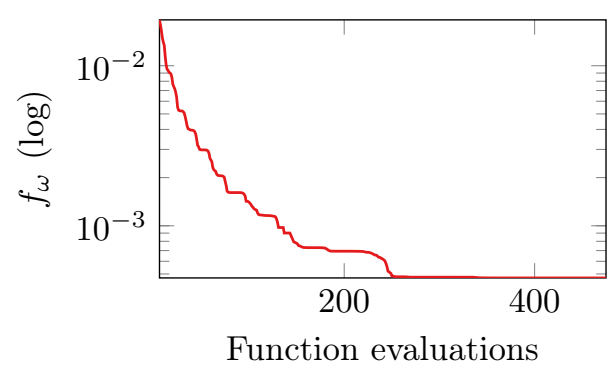

(b) L-BFGS-B

FIG. 3. Box-contrained minimization of the functional $f_{\omega}$ using projected gradient descent and $L-B F G S-B$ respectively. The setup is taken from the original publication [36], using the 16-degree ZoLOTAREV filter as starting point and a lower bound of $l b=0.0022$ on the absolute value of the imaginary parts of the poles. The L-BFGS-B method settles at a smaller residual and converges substantially faster, requiring only a few function evaluations only.

experiments, when compared with projected gradient descent. In terms of both speed and accuracy, the use of L-BFGS-B places the constrained SLiSe method on par with the unconstrained BFGS algorithm. To illustrate the increase in performance caused by L-BFGS-B, we compare box-constrained minimization through our L-BFGS-B implementation against the projected gradient descent implemented in the original SLiSe framework. Figures 3a and $3 \mathrm{~b}$ show the number of function evaluations carried out by the projected gradient descent and the L-BFGS-B algorithms, respectively. L-BFGS-B requires four order of magnitudes fewer evaluations than projected gradient descent, and converges to a smaller residual.

So far, the procedure used to obtain the SLiSe filters depends on the specific form of a given weight function $\omega$. For some of such weight functions, the outputted filters were shown to outperform state-of-the-art rational filters. Yet, the only criterion known to determine suitable weight functions is by comparing hand-crafted weight functions on a large set of representative interior eigenproblems. While guidelines for the construction of $\omega$ have been devised, the choice of weight functions remains a complex issue. In the following section, we propose an algorithm to obtain weight functions, which yield SLiSe filters with reduced WCR, and overcome the necessity of selecting weight functions manually.

4. SLiSe filters with reduced WCR. In this section, we illustrate how to reduce the WCR of a given SLiSe filter by improving on the choice of weight function $\omega$. We achieve this by minimizing a new objective function, closely related to the WCR of rational filters.

4.1. Parameterization of weight functions. Weight functions are even, nonnegative, piecewise constant functions that are used in the definition of the SLiSe functional $f_{\omega}$ in Eq. (1.3). This means that a weight function can be characterized in terms of $n \in \mathbb{N}$ intervals $\left[x_{i}, x_{i+1}\right) \subseteq[0, \infty]$ and corresponding function values $\omega\left(x \in\left[x_{i}, x_{i+1}\right)\right)=\omega_{i}$, called weights, where $\omega_{i} \in[0, \infty)$ for $i=1,2, \ldots, n$. In their original contribution, Winkelmann et al. obtained weight functions for the SLiSe framework by following three guiding principles, derived from experience: (i) gradual decrease in weights outside the search interval $[-1,1]$, (ii) sufficient magnitude of weights inside $[-1,1]$, and (iii) symmetry in weights about the interval endpoints of $[-1,1]$. While SLiSe filters following these guidelines could outperform state-of-the- 


\begin{tabular}{lccccc}
\hline$|x| \in$ & {$[0,0.95)$} & {$[0.95,1.05)$} & {$[1.05,1.4)$} & {$[1.4,5)$} & {$[5, \infty)$} \\
\hline$\omega_{\gamma \text {-SLiSe }}(x)$ & 1 & 0.01 & 10 & 20 & 0 \\
\hline
\end{tabular}

TABLE 1

The $\omega_{\gamma-S L i S e}$ weight function

\begin{tabular}{cccccc}
\hline$|x| \in$ & {$\left[0, v_{1}\right)$} & {$\left[v_{1}, v_{2}\right)$} & {$\left[v_{2}, v_{3}\right)$} & {$\left[v_{3}, v_{4}\right)$} & {$\left[v_{4}, \infty\right)$} \\
\hline$\omega_{j}(x)$ & 1 & $v_{5}$ & $v_{6}$ & $v_{7}$ & 0 \\
\hline
\end{tabular}

TABLE 2

Parameterized weight function $\omega$, for $s=5$.

art Gauss-Legendre and Zolotarev filters, some manual adjustment based on experience remained necessary.

An example of a weight function $\omega_{\gamma \text {-SLiSe }}$, which yields SLiSe filters outperforming Gauss-Legendre filters, is given in Table 1. This choice of weights suggests a natural way of parameterizing weights and interval boundaries, so they can be treated without distinction. For this purpose, we introduce a set $V_{s}$ of parameter vectors $v=\left(v_{1}, \ldots, v_{2 s-3}\right)$, where $s \geq 2$ equals the number of intervals to the right of 0

$$
V_{s}=\left\{v \in[0, \infty)^{2 s-3} \mid G<v_{1}<1<G^{-1}<v_{2}<v_{3}<\ldots<v_{s-1}\right\},
$$

for some gap parameter $G \in(0,1)$.

A generic set of $v_{i} \in V_{s}$ induces a weight function $\omega_{j}$ with $j=1,2, \ldots, s$. Following this parameterization, Table 1 is rewritten as Table 2 . The parameters $v_{1}, v_{2}$ enclose \pm 1 , but do not necessarily match the endpoints of the gap $\left[G, G^{-1}\right]$. The parameters $v_{3}, v_{4}$ reflect some more intervals of the weight function. The remaining parameters $v_{5}, \ldots, v_{7}$ denote non-negative weights. The weight for the interval $\left[0, v_{1}\right)$ is fixed to 1 , as weight functions are invariant under scaling within SLiSe. In this notation, the $\omega_{\gamma \text {-SLiSe weight }}$ function from Table 1 translates into the vector $(0.95,1.05,1.4,5,0.01,10,20) \in V_{5}$. As we are going to illustrate in the next section, this parameterization scheme allows for a systematic improvement of weight functions, alongside a choice of weights and interval endings.

4.2. Minimization of parameterized weight functions. In order to compare the influence of distinct weight functions $\omega_{j} \in V_{s}$ on the minimization of WCR, we introduce a new objective function

$$
h:=h_{\beta, z}(v):=w_{G}\left(r_{\beta, z}(v)\right), \quad \text { for } v \in V_{s},
$$

where $r_{\beta, z}(v)$ is computed by the Algorithm 3.1. $h_{\beta, z}(v)$ is a functional of a given filter $r_{\beta, z}$, and it associates the vector of parameters $v$ with the WCR of the corresponding filter. As such, $h$ establishes a meaningful metric to quantify the performance of a weight function $\omega$. The minimization

$$
\underset{v \in V_{s}}{\operatorname{argmin}} h_{\beta, z}(v),
$$

facilitates a systematic search for better weight functions and, consequently, rational filters with smaller worst-case convergence. For a given $r_{\beta, z}$, this is a non-linear, 


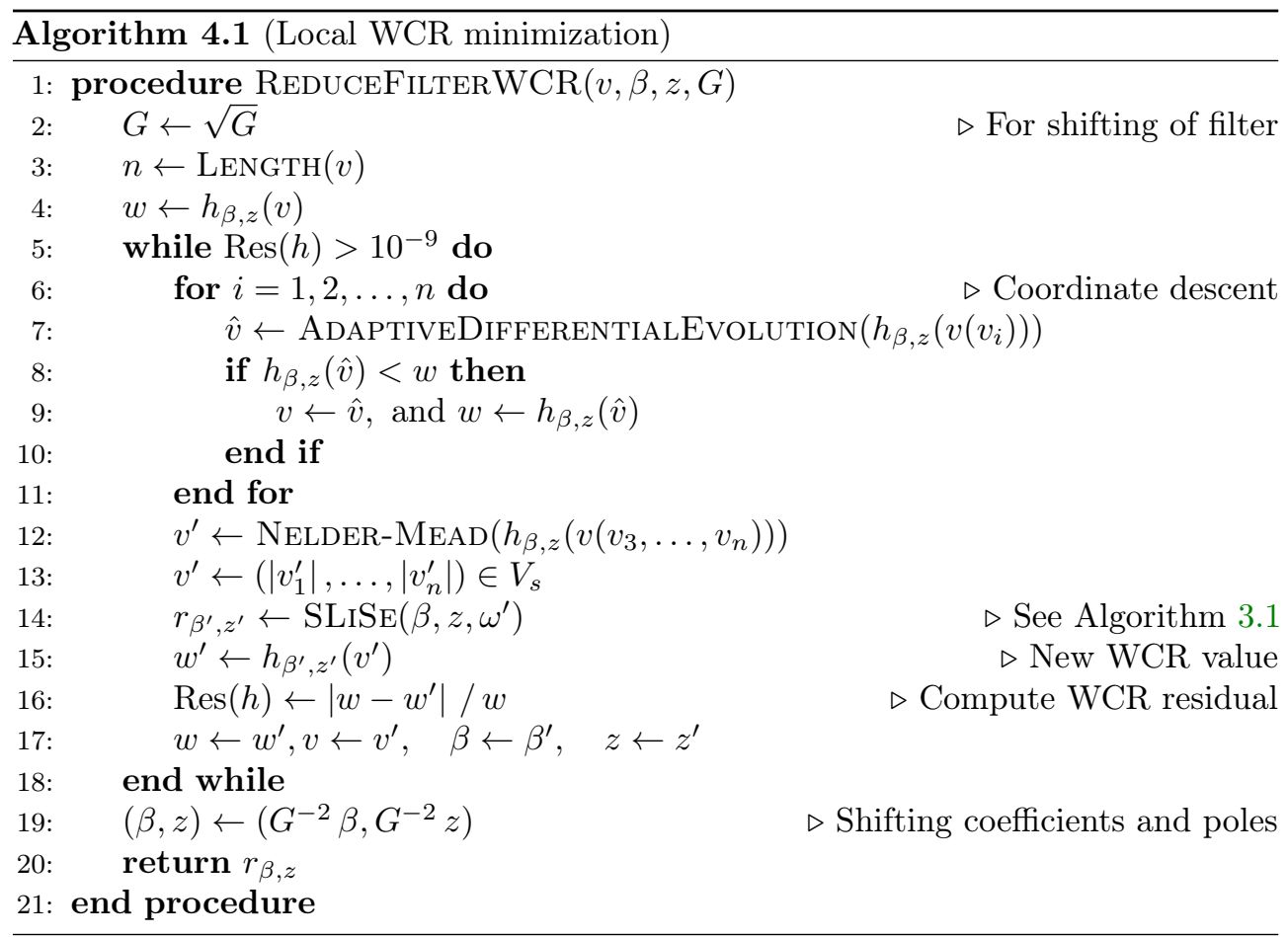

derivative-free minimization problem, depending on only $(2 s-3)$ variables. However, for each optimization step involving changes of $v$, the rational filter $r_{\beta, z}(v)$ has to be computed again by executing a call to the Algorithm 3.1. The end result is a nested optimization problem Eq. (2.8) with, possibly, thousands of calls to Algorithm 3.1.

Since the WCR functional cannot be expressed as a continuous function of $v$, to solve Eq. (4.3), we resort to using the NELDER-MEAD algorithm, a prominent local, derivative-free minimization schemes. ${ }^{3}$ In our case, NELDER-MEAD generates competitive solutions quickly, but suffers from stagnation at non-optimal points [23, 20]. To overcome stagnation, we follow Carl Kelley's suggestion of restarting NELDERMEAD at the current iterate with adjusted parameters [17]. An explicit such parameter choice exists only for the case of smooth functions, introduced as oriented restart. Since our functional $h$ is non-smooth, we obtain a new parameter choice by perturbing the current iterate carefully through coordinate descent. Coordinate descent is a simple, local, derivative-free minimization method, that performs subsequent line searches along the coordinate directions, given some starting point $v \in V_{s}$. Independently of having detected stagnation, we use coordinate descent systematically to obtain a new starting point for each NELDER-MEAD call.

The general scheme outlined above is described in Algorithm 4.1 and implemented using the Julia programming language. ${ }^{4}$ Given a weight function $v \in V_{s}$ and a filter $r_{\beta, z}$, the algorithm chooses better weight functions from $V_{s}$ iteratively. At each iteration of the while loop, the weights of the current filter are updated to reduce the WCR, and from these, a new filter is computed. When the residual of the $h$

\footnotetext{
${ }^{3}$ Local means that the algorithm starts at an existing point, at best, close to the sought after minimum.

${ }^{4}$ The code is freely available at https://github.com/SimLabQuantumMaterials/SLiSeFilters.jl
} 
function $\operatorname{Res}(h)$ (i.e. the relative difference of two subsequent $h$ values) falls below an established threshold tolerance (see line 5 of Algorithm 4.1), the algorithm returns the SLiSe filter of the last iteration, which minimizes the WCR among the weight functions in the search space $V_{s}$. Each while loop iteration follows three consecutive steps: (i) coordinate descent, (ii) NELDER-MEAD, (iii) computation of new SLiSe filter and convergence check.

Coordinate descent. This is performed in Lines 6 - 11, improving the coordinates of the parameter vector $v \in V_{s}$ through a separate minimization problem for each variable $v_{i}$ and $i \leq 2 s-3$,

$$
\underset{c \in I_{i}}{\operatorname{argmin}} h_{\beta, z}(v(c)), \quad \text { where } v(c):=\left(v_{1}, \ldots v_{i-1}, c, v_{i+1} \ldots, v_{2 s-3}\right) \in V_{s},
$$

while restricting the search space to a neighborhood $I_{i}$ of $v_{i}$. For instance, for $s \geq 5$ the intervals $I_{i}$ used are

$$
I_{i}:= \begin{cases}{[G, \quad 1],} & \text { if } i=1 \\ {\left[1, \quad G^{-1}\right],} & \text { if } i=2 \\ {\left[G^{-1}, v_{i+1}\right],} & \text { if } i=3 \\ {\left[v_{i-1}, v_{i+1}\right],} & \text { if } 4 \leq i<s-1, \\ {\left[v_{i-1}, 3 v_{i}\right],} & \text { if } i=s-1 \\ {\left[0.1 v_{i}, 10 v_{i}\right],} & \text { if } s \leq i \leq 2 s-3 .\end{cases}
$$

We implement the coordinate descent minimization through the global, derivative-free minimization scheme Adaptive Differential Evolution from the Julia library BlackBoxOptim.jl [7]. Global minimization algorithms aim to find the global minimizer within a region $I_{i}$, instead of converging to a local minimizer starting from a given point. If the value of WCR has decreased, the solution of the coordinate descent minimization $\hat{v}_{i}$ is used instead of $v_{i}$ for the successive steps of the while loop iteration. This step is executed to prevent stagnation in the execution of the NELDEAR-MEAD minimization.

Nelder-Mead. In Line 12, NELDER-MEAD is applied to a slightly modified version of the minimization problem formulated in Eq. (4.3): We keep $v_{1}, v_{2}$ fixed at the values obtained from the coordinate descent step. This choice is motivated by the high sensitivity of the functional $h$ to changes in these variables, being close to the endpoints of the gap $\left[G, G^{-1}\right]$. In practice, NELDER-MEAD is used to solve the minimization problem

$$
\underset{b_{1}, \ldots, b_{r} \in \mathbb{R}}{\operatorname{argmin}} h_{\beta, z}\left(v\left(b_{1}, \ldots, b_{r}\right)\right),
$$

where

$$
v\left(b_{1}, \ldots, b_{r}\right):=\left(v_{1}, v_{2}, b_{1}, \ldots, b_{r}\right) \text { and } \quad r=2 s-5 .
$$

Because the classical NELDER-MEAD algorithm performs unconstrained minimization only, we also have to ensure that the minimizer $v^{\prime}$ lies within the admissible intervals defined by $V_{s}$. A straightforward approach would be to use a modified objective function within NELDER-MEAD, defined as

$$
\hat{h}(v):= \begin{cases}h(v), & \text { if } v \in V_{s}, \\ \infty, & \text { otherwise }\end{cases}
$$




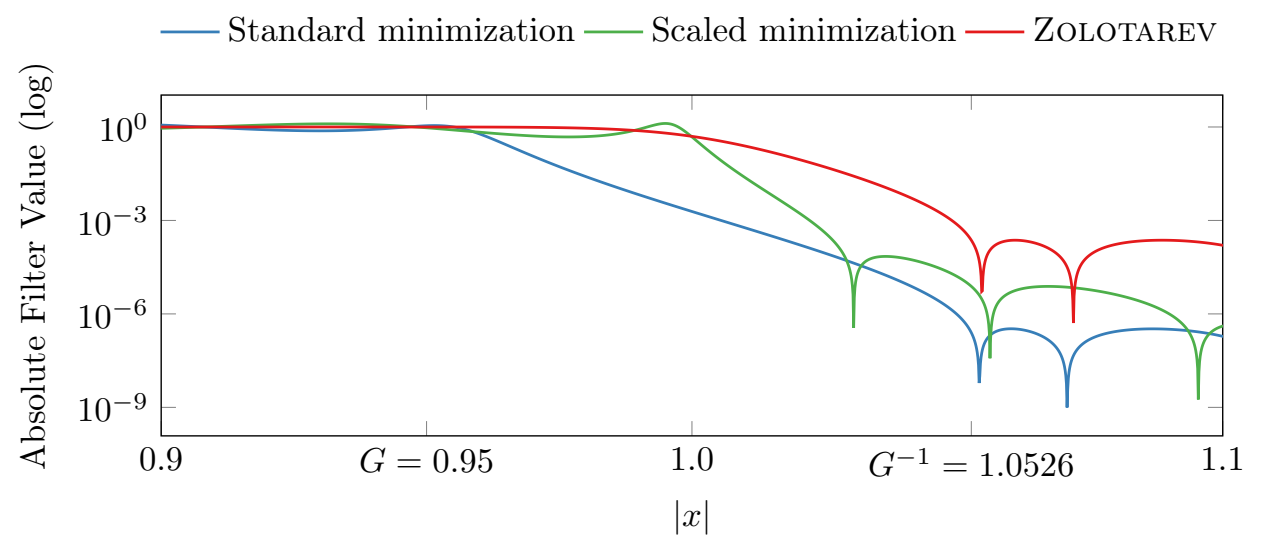

FIG. 4. Logarithmic plot of 16-pole rational filters for gap parameter $G=0.95$. The standard filter is a solution of the original minimization problem as of Eq. (4.3), while the scaled filter solves the new minimization problem in Eq. (4.7). Even though the scaling causes the WCR to increase from $4.95 \mathrm{e}-6$ to $1.04 \mathrm{e}-5$, this is still below the WCR of ZOLOTAREV filter (2.32e-4). On the other hand, near 1 , the standard filter attains a function value of about $10^{-3}$. Hence, an eigenvalue inside the gap $[G, 1]$ could roughly half the actual convergence rate for the standard filter. The other two filters are not affected by this problem.

to penalize invalid weight functions $v \notin V_{s}$. We verified that this approach works, but our experiments have shown that it slows down the convergence to the minimum. Based on our tests, we observed that, in practice, most of the violations $v \notin V_{s}$ stem from the selection of slightly negative weights by NELDER-MEAD. This is likely caused by rounding errors, which may lead to a slight decrease of the WCR value for very small but negative $\omega_{j}$. Every other violation of constraints seems to cause the opposite of a reduction in WCR value and is thus not chosen by NELDER-MEAD. To overcome this problem, we adopted a very simple solution: We map the resulting minimizer of a NELDER-MEAD into $V_{s}$ explicitly, by taking the absolute values $\left(\left|v_{1}^{(k+1)}\right|, \ldots,\left|v_{2 s-3}^{(k+1)}\right|\right)$ instead of a possible negative (invalid) iterate $v^{(k+1)}$ (see line 13). While this approach only avoids violations of constraints caused by the choice of negative weights, we did not experience other violations of constraints in the minimizers returned by NELDERMEAD. For the sake of completeness, we have experimented with other derivative-free minimization algorithms (those from the minimization algorithm collection NLOpt [16], including a constrained version of NELDER-MEAD), none of which led to a more competitive reduction of WCR value in the same standard setups.

SLiSe filter and convergence. As already mentioned, the SLiSe procedure is called multiple times within both the NELDER-MeAd and the AdAPtiveDifferentialEvoLUTION procedures. The last call of SLiSe is executed so as to calculate the residual of the WCR functional and check for convergence. If convergence is reached, the algorithm returns values for the WCR, the poles $z$ and the coefficients $\beta$. The latter are rescaled, as it is explained in the following Subsection 4.3, by introducing a linear scaling transformation to improve the behavior of the resulting filters at the interval $[-1,1]$ boundaries.

4.3. Scaling the filter. Compared to previous filters such as Gauss-Legendre or ZolotaRev, our new SLiSe filters obtained from the minimization problem Eq. (4.3) reduce the WCR by up to multiple orders of magnitude. So far, we assumed to know 
the appropriate gap parameter $G$ in advance and minimized our filters accordingly. This assumption may be too optimistic for some eigenproblems: For a given $G$, we assume that no eigenvalues lie within the interval $\left[-G^{-1},-G\right] \cup\left[G, G^{-1}\right]$. Since, in practice, this assumption may be violated, a rational filter with small function value within $[G, 1]$ may lead a slower convergence rate than the WCR, or no convergence at all. An illustration of this problem is given in Figure 4, and its caption. Eigenvalues within $\left[1, G^{-1}\right]$ are less problematic because they are not sought after by the eigensolver (for an in-depth discussion, see Section 5.2). While maintaining a competitive WCR value, the issue described above can be overcome by solving the slightly modified minimization problem

$$
\underset{\beta \in \mathbb{C}^{m}, z \in(\mathbb{C} \backslash \mathbb{R})^{m}}{\operatorname{argmin}} \frac{\max _{x \in\left[-\infty,-G^{-1}\right] \cup\left[G^{-1}, \infty\right]}\left|r_{\beta, z}(x)\right|}{\min _{x \in[-1,1]}\left|r_{\beta, z}(x)\right|}
$$

instead of minimizing $w_{G}$ as in Eq. (2.7). For any solution $(\beta, z)$ of this modified problem, the rational filter $r_{\beta, z}$ offers a larger function value inside the entire search interval $[-1,1]$ than outside in $\left[-\infty,-G^{-1}\right] \cup\left[G^{-1}, \infty\right]$. When our filters are used in iterative eigensolvers based on spectral projection, this behavior ensures reliable and fast convergence.

Instead of modifying the WCR minimization procedure, we solve for the modified WCR in Eq. (4.7) by introducing a linear scaling transformation $u(x):=\sqrt{G} x$. We have

$$
\underset{\beta \in \mathbb{C}^{m}, z \in\left(\mathbb{H}^{+R}\right)^{m}}{\operatorname{argmin}} w_{\sqrt{G}}\left(r_{\beta, z}\right)=\underset{\beta \in \mathbb{C}^{m}, z \in\left(\mathbb{H}^{+R}\right)^{m}}{\operatorname{argmin}} \frac{\max _{x \in\left[-\infty,-G^{-1}\right] \cup\left[G^{-1}, \infty\right]}\left|r_{\beta, z}(u(x))\right|}{\min _{x \in[-1,1]}\left|r_{\beta, z}(u(x))\right|} .
$$

Since the function composition $r_{\beta, z} \circ u$ is a rational filter itself, if $r_{\beta, z}$ solves Eq. (4.3), then $r_{\beta, z} \circ u$ solves Eq. (4.7). Additionally, it follows from the definition of rational filters in Eq. (1.2) that $r_{\beta, z} \circ u=r_{\sqrt{G^{-1}}} \beta, \sqrt{G^{-1}} z$ which characterizes the parameters of the resulting filter. This scaling of rational filters through linear transformation is incorporated in Algorithm 4.1 in lines 2 and 19.

In our implementation of Algorithm 4.1, we used as initial parameters the weight functions $\omega_{\gamma \text {-SLiSe }}$ used in [36] opportunely rescaled. For instance, for $s=5$, we selected

$$
v^{(0)}=\left(\sqrt{G}, \sqrt{G^{-1}}, 1.4,5, .01,10,20\right)
$$

as the initial parameters characterizing the weight function, for some gap parameter $G \in(0,1)$. Accordingly, we chose both Zolotarev and Gauss-Legendre filters as initial conditions for WCR minimization. We obtained a number of new spectral filters, to which we refer as Worst-Case Optimized Least-Squares (WiSe). In the following section, we provide a number of experimental tests illustrating the performance of these WiSe filters.

5. Experiments. In this section, we compare the ZOLOTAREV and generalized GAUSS-LEGENDRE filters to the WiSe filters from the previous section in two different scenarios. First, in order to inspect the worst-case performance, we compute the WCR values for different gap parameters $G$ and poles per quadrant $m$. Second, we use the filters in the FEAST package and assess their performance on two eigenproblems used in past literature [31, 36, 12].

We provide the Julia library SLiSeFilters.jl to obtain WiSe filters and the generalized Gauss-Legendre filters. For Zolotarev filters, we use the RKToolbox by 


\begin{tabular}{|c|c|c|c|c|}
\hline Gap $G$ & $\begin{array}{l}\text { Poles per } \\
\text { Quadrant } m\end{array}$ & $\begin{array}{c}\text { GAUSS- } \\
\text { LEGENDRE }\end{array}$ & ZOLOTAREV & WiSe \\
\hline \multirow[t]{5}{*}{0.95} & 3 & $2.33 e-2$ & $2.24 e-3$ & $2.45 e-4$ \\
\hline & 4 & $1.37 e-3$ & $2.32 e-4$ & $1.04 e-5$ \\
\hline & 5 & $5.58 e-4$ & $2.41 e-5$ & $9.82 e-7$ \\
\hline & 6 & $4.80 e-5$ & $2.50 e-6$ & $7.53 e-7$ \\
\hline & 7 & $7.36 e-6$ & $2.59 e-7$ & $2.73 e-7$ \\
\hline \multirow[t]{5}{*}{0.98} & 3 & $3.45 e-2$ & $7.46 e-3$ & $1.30 e-3$ \\
\hline & 4 & $2.43 e-2$ & $1.15 e-3$ & $1.63 e-4$ \\
\hline & 5 & $1.94 e-3$ & $1.77 e-4$ & $1.47 e-5$ \\
\hline & 6 & $1.16 e-3$ & $2.74 e-5$ & $1.56 e-5$ \\
\hline & 7 & $1.01 e-4$ & $4.24 e-6$ & $9.52 e-6$ \\
\hline \multirow[t]{5}{*}{0.998} & 3 & $6.64 e-1$ & $4.23 e-2$ & $2.74 e-2$ \\
\hline & 4 & $4.69 e-1$ & $1.12 e-2$ & $3.53 e-3$ \\
\hline & 5 & $8.29 e-2$ & $3.04 e-3$ & $7.33 e-4$ \\
\hline & 6 & $4.45 e-2$ & $8.27 e-4$ & $9.81 e-5$ \\
\hline & 7 & $3.15 e-2$ & $2.26 e-4$ & $8.94 e-6$ \\
\hline \multirow[t]{5}{*}{0.9998} & 3 & $9.51 e-1$ & $1.11 \mathrm{e}-1$ & $1.44 e-1$ \\
\hline & 4 & $8.97 e-1$ & $3.85 e-2$ & $4.16 e-2$ \\
\hline & 5 & $8.22 e-1$ & $1.39 e-2$ & $1.04 \mathrm{e}-2$ \\
\hline & 6 & $7.35 e-1$ & $5.09 e-3$ & $1.85 e-3$ \\
\hline & 7 & $6.41 e-1$ & $1.87 e-3$ & $5.19 e-4$ \\
\hline
\end{tabular}

WCR values for different filters, gap parameters, and numbers of poles (smaller is better; row-wise minimum in bold).

Güttel et al. We will not consider other prominent examples of rational filters, notably Trapezoid or former SLiSe filters, as they are not as competitive. Trapezoid filters offer a strictly monotonous decay in function value for $|x|>1$, which is not as sharp as for GAuss-LEgENDRE and ZolotaRev filters leading to significantly larger WCR values [12]. As for SLiSe filters, Figure 1 provides clear evidence that WiSe filters are superior when it comes to number of iterations to convergence.

5.1. Comparison of WCR values. In Table 3, we list WCR values for GaussLEGENDRE, ZOLOTAREV, and WiSe filters for different gap parameters $G$ and poles per quadrant $m$. As $m$ increases, ZolotAREv filters feature a reliable, gradual decrease in WCR; by construction, they do not offer a decay in function value as $|x| \rightarrow \infty$, unlike Gauss-Legendre and WiSe. Hence, Gauss-Legendre and WiSe filters lead to quicker convergence for some FEAST instances, even if their WCR is larger. GAUSS-LEGENDRE filters show the largest WCRs and are not competitive with regards to worst-case performance. WiSe exhibit a significant reduction of WCR compared to previous filters, especially for the default choice of $m=4$ within the FEAST eigensolver. Yet, the improvement over ZolotaRev filters diminishes as $m$ increases, because the dimension of the underlying minimization increases with $m$. Large numbers of poles per quadrant $m>7$ correspond to high-degree rational functions and are not taken into consideration. It is important to notice that WiSe filters seem to perform particularly well for large $G$ and $m$, making them especially competitive in the presence of eigenvalue clusters near the interval boundaries.

The numbers in bold in Table 3 show that, for almost all pairs $(G, m)$, WiSe filters have the lowest WCR value. Based on Theorem 2.1, the best worst-case performance is offered by the filter with smallest WCR. As we will see in the following sections, this claim is confirmed by our numerical results.

5.2. Experiments with FEAST. For our numerical experiments, we used FEAST in the version 3.0, compiled with the Intel Compiler 17.0.0, and run on an Intel Core i7-6900K. We selected the default FEAST parameters, with the exception of dis- 
abling $^{5}$ the repeated factorization of the underlying linear systems. This substantially reduces runtime, but requires sufficient RAM.

A required argument of FEAST is an upper bound $M_{0}$ on the number of eigenvalues $M$ in the search interval; $M_{0}$ indicates the size of the reduced eigenvalue problem, that is solved in every FEAST iteration. As already mentioned in Sec. 2, the convergence rate of FEAST substantially depends on this subspace size $M_{0}$. Let us denote with $I \supset[-1,1]$ the interval centered around 0 that contains $M_{0}>M$ eigenvalues; then, FEAST's convergence rate is proportional to

$$
\frac{\left|r\left(\lambda_{\text {out }}\right)\right|}{\left|r\left(\lambda_{\text {in }}\right)\right|}
$$

where $\left|r\left(\lambda_{\text {in }}\right)\right|=\min _{\lambda \in[-1,1] \cap \sigma(A)}|r(\lambda)|$, and $\left|r\left(\lambda_{\text {out }}\right)\right|=\max _{\lambda \notin I \cap \sigma(A)}|r(\lambda)|$. A smaller subspace size $M_{0}$ yields faster FEAST iterations, but decreases the convergence rate and thus increases the number of iterations. As a compromise, the original FEAST publication [31] suggested a subspace size of $M_{0}=\lceil C \times M\rceil$, for $C=1.5$. This provides reliable convergence within FEAST, but not necessarily fastest convergence as we see in the following. Because only estimates of the actual eigencounts are available in advance, we assess different scenarios by studying a number of eigencount multipliers $C$.

It is important to notice that in all our comparison we change only the rational filter and maintain all other part of FEAST unchanged. In particular we let FEAST use the same default linear system solves to tackle Eq. (2.3) and direct eigensolver in the Rayleigh-Ritz step. Since the level of accuracy of FEAST is for all practical purposes determined by these two tasks [18, 19], we consider the accuracy of the determined eigenpairs across distinct filters comparable. In other words, since FEAST reaches convergence with all filters using the same procedures, then the solution are considered to be equally accurate.

5.2.1. Experiment I. In Figure 5, we compare the convergence of FEAST for different eigencount multipliers $C>1$ on a specific interval of the so-called CNT eigenproblem. The matrices, corresponding to this generalized eigenproblem, represent the discretized Hamiltonian and Overlap operators of a physical system studied in the context of a specific Density Functional Theory method and have been used to analyze the worst-case performance of FEAST in previous publications [31, 12]. These sparse CNT matrices $A, B \in \mathbb{R}^{12450 \times 12450}$, with 86808 non-zero entries, define the interior eigenproblem $A x=\lambda B x$, where one is interested in obtaining the $M=100$ eigenvalues in the interval $[-65.0,4.96]$. The figure is divided into three quadrants, each corresponding to one of three increasing eigencount multipliers $C=1.02,1.1,1.5$. On the $\mathrm{x}$-axis of each quadrant, the convergence time (in seconds) of the FEAST eigensolver, equipped with three distinct filters, is plotted against increasing numbers of poles per quadrant $m$ for each of these filters. The value of the parameter $G$ is kept fixed for all filters across the entire figure.

The result of this experiment demonstrates that best worst-case convergence of the FEAST eigensolver correlates strongly with the WCR value of the filter that is used to project onto the active subspace. On the other hand, the size of the active subspace $M_{0}$ also contributes to the convergence time and can be a confounding factor in interpreting the numerical results. In order to minimize the influence of the latter

\footnotetext{
${ }^{5}$ This behavior can be achieved through the FEAST parameter $\mathrm{fpm}(10)=1$. For details, consult the FEAST documentation [6].
} 
—Gauss-Legendre $0.998 \_$Z Zolotarev 0.998 - WiSe 0.998

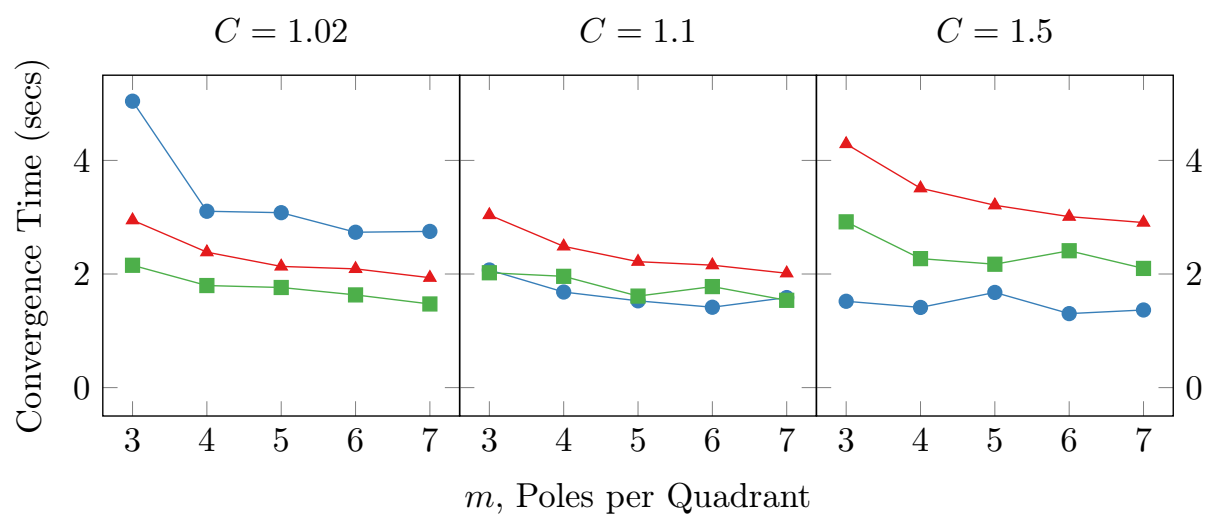

FiG. 5. Time required to solve the CNT eigenproblem [31, 12] through FEAST, for different eigencount multipliers $C$, numbers of poles, and filters. Averages of 10 executions are reported. All the three filters lead to eigenpairs with the same level of accuracy.

on our interpretation of WCR, we consider first the quadrant for $C=1.02$. From Table 3, we expect that best convergence time is achieved by FEAST when equipped with WiSe filters, followed by ZOLOTAREv and GAuss-LegendRe filters, respectively. This is indeed the case; the performance of the three filters is clearly separated by a gap, which reflects the differences in WCR between the filters for all $m$.

The influence of $m$ on filter performance is less pronounced. This is not surprising. For instance, the ZoLOTAREv filter enables FEAST to converge with a very slow decrease of convergence rate as the number of poles increases, for all considered $C$. The only consequence of increasing $C$ is a growth in convergence time, since the size of the subspace increases with $C$ and more operations with vectors must be performed by the linear system solver. This behavior is due to the so-called equi-oscillation of ZOLOTAREV filters: These filters are optimal in approximating the ideal filter in $\infty$-norm, but do not decay away from the filtered interval. WiSe filters behave similarly: While they decay (moderately) away from the interval, their effectiveness is not determined by their value away from it but rather their behavior very close to its boundary (see Figure 2). This is reflected by the very slight decrease in time-to-convergence as $m$ increases although the corresponding WCR value decreases substantially as shown in Table 3.

As the size of the active subspace increases, its influence on convergence time becomes ever more pronounced. This is because the difference between the convergence rate of FEAST in Eq. (5.1) may become increasingly larger than the WCR of the used filter as $C \gg 1.02$ [12]. In other words, larger active subspaces dilute the correlation between worst-case convergence rate of the filter and convergence rate of the eigensolver. This is clearly visible, if one traverses the quadrants in Figure 5 from left to right, and is best illustrated by the GaUss-LEgENDRE filters. These suffer from slow convergence for small $C$, but can compensate for their large WCR for large subspace sizes because they decay rapidly in function value away from the search interval. In other words, for GAUSS-LEGENDRE, the size of the active subspace is much more relevant than the WCR of the filter.

This simple analysis, based on a very specific interior eigenvalue problem, seems 


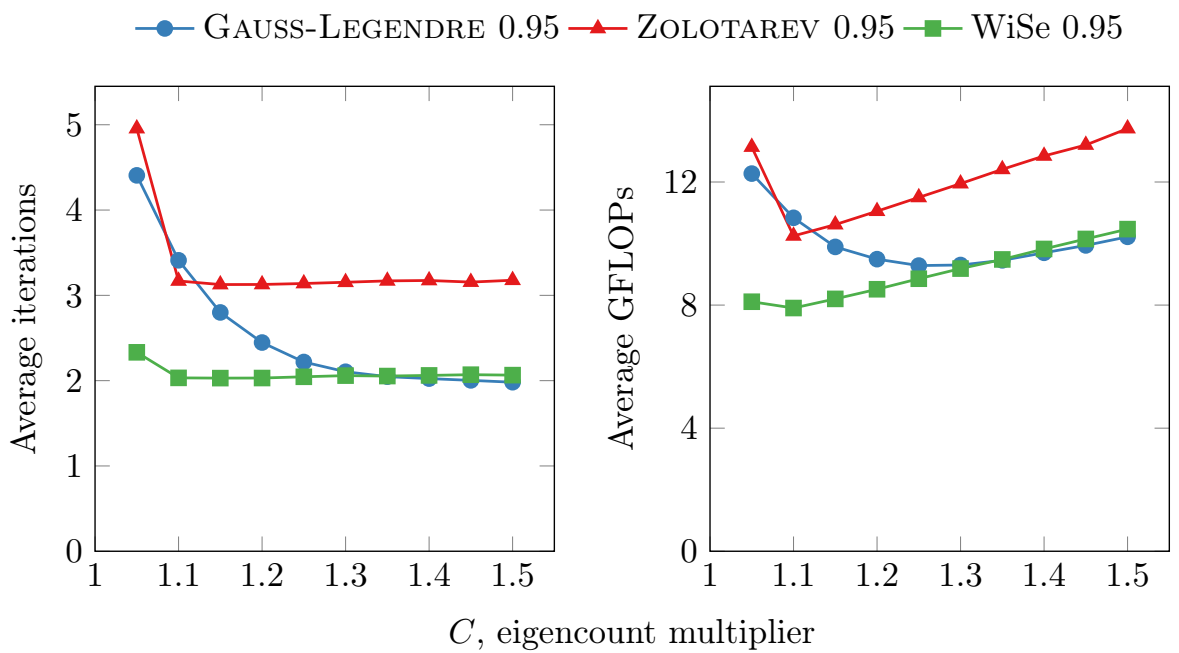

FIG. 6. The average numbers of iterations and FLOPs (floating-point operations), required by FEAST to solve 2117 benchmark problems [36], for different subspace sizes (by multiplying the actual eigencounts with a fixed scalar $C$ ) and filters with $G=0.95$.

to suggest that WiSe filters should always be preferred over ZOLOTAREv filters in those cases in which the WiSe WCR is smaller than ZoLOTAREV (compare Table 3). When comparing the performance of WiSe and Gauss-LEgEndRE filters, it seems that the size of the active subspace plays a major role in identifying the point at which one filter outperforms the other. In order to address this question, we examine the convergence rate of FEAST in the next section, both in terms of number of FLOPs and number of subspace iterations, using a large set of representative eigenproblems.

5.2.2. Experiment II. We consider a moderate gap parameter of $G=0.95$ and a set of 2117 interior eigenproblems. These eigenproblems were obtained from $\mathrm{Si2}$, a sparse and symmetric matrix from the University of Florida Matrix Collection [4], by selecting 2117 different search intervals $[a, b]^{6}$ as described in [36, Appendix B]. Each search interval uniquely identifies an interior eigenproblem with its unique eigenvalue distribution and eigenvalue count. As such, it is quite general and statistically relevant, since it reflects the large variations that are possible in distributing and clustering eigenvalues inside, outside, and in the vicinity of the search interval ends.

We initially solved for each of the 2117 benchmark problems with a fixed value of $m=4$ poles per quadrant, the default in the FEAST eigensolver. As in the previous section, we repeated this test for all three filters for increasing values of the eigencount multiplier $C$. The results of these tests are graphically reported in Figure 6, which plots the number of subspace iterations and the total number of FLOPs performed by FEAST. These results confirm the analysis of Section 5.2.1. The ZOLOTAREV and WiSe filters maintain a linear behavior as a function of increasing dimension of the active subspace as soon as $C \geq 1.1$. In other words, the WCR of these filters influences the convergence of the eigensolver only for active subspaces that closely match the true number of eigenvalues in the interval $[a, b]$. As soon as the size of the active

\footnotetext{
${ }^{6}$ The code to obtain such benchmark sets from arbitrary matrices is freely available at https: //github.com/SimLabQuantumMaterials/SpectrumSlicingTestSuite.jl.
} 


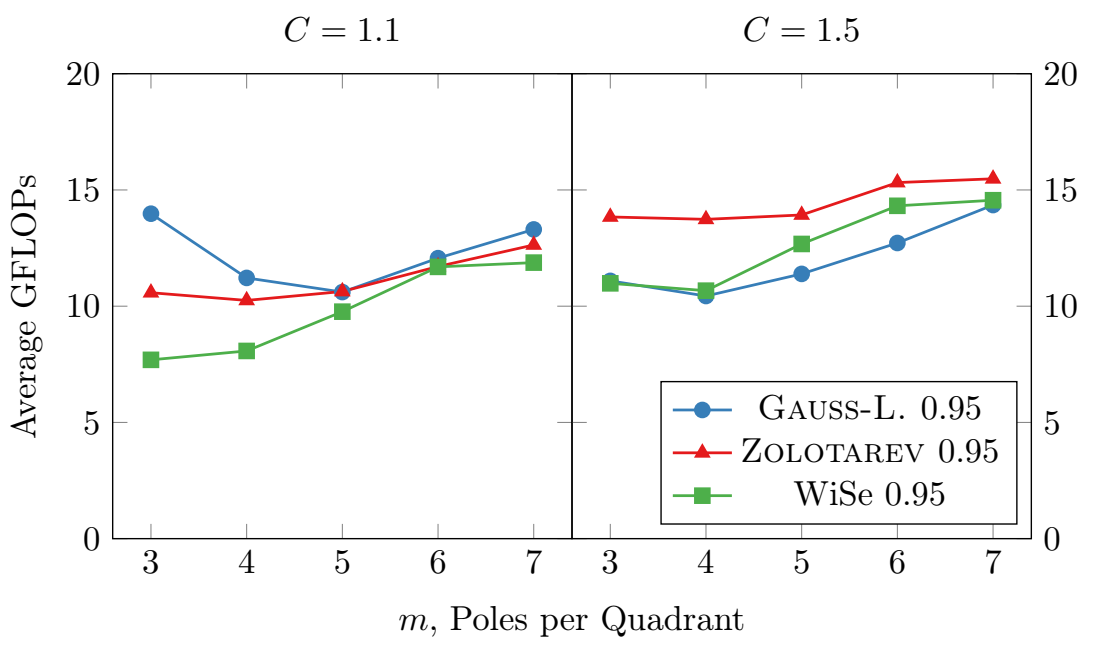

FIG. 7. Comparison of average FLOPs, required to solve 2117 benchmark problems [36], using Gauss-Legendre, Zolotarev, and WiSe filters for different of poles numbers $m$ per quadrant, while $C \in\{1.1,1.5\}$ and $G=0.95$.

subspace gets larger, the convergence of the eigensolver is dictated by Eq. (5.1). This interpretation is made even clearer when one looks at the linear increase in FLOPs for FEAST, equipped with these two filters: While the average number of iterations remains constant, the total number of floating-point operations increases due to the linear increase in the total number of right-hand-side vectors $Y$ for which Eq. (2.3) must be solved.

As $C$ grows, the rate of convergence of FEAST, equipped with the GAussLEGENDRE filter, equals the one of WiSe - which is dictated by the WCR at smaller values of $C$. For subspace multipliers $C>1.3$, the GaUss-LEgENDRE starts competing, on average, with the WiSe. It must be noted that this comes at a cost: Using GaussLEGENDRE for a relatively large active subspace, such as the default value of $C=1.5$ suggested by FEAST, has on average a higher FLOP count than the WiSe filters for eigenvalue counts $C<1.3$. This observation is fairly independent from the number of poles per quadrant used, as shown in Figure 7. Gauss-LegendRe filters have a slight advantage with respect to FLOP count for large subspace sizes $(C=1.5)$, but they behave worse than the WiSe for any number of poles and small subspace sizes $(C=1.1)$. Due to the decay in value of the filter function, the WiSe filter even outperforms ZoLOTAREv for $m=7$, despite its larger WCR. For larger $m$, overall FLOP count increases, while the differences in FLOP count across the filters shrinks.

In conclusion, FEAST, equipped with WiSe filters, offers a competitive advantage over the use of Gauss-Legendre and Zolotarev filters. WiSe filters are quite stable with respect to the convergence rate of the eigensolver, irrespective of the active subspace or the degree of the filter function. Their use seems to almost always minimize the total FLOP count required by FEAST to reach convergence. In addition, their effectiveness for small eigencount multipliers suggests that WiSe filters should be preferred in all those cases where it is necessary to contain the subspace size, either because the RAM is limited or the underlying spectrum distribution is unknown.

6. Conclusions. In this work, we show how we decreased time to convergence of the SLiSe optimization framework by using in it the minimization algorithm L- 
BFGS-B. When computing a box-constrained SLiSe filter, only hundreds of function evaluations are needed instead of millions. We exploit the improved performance by introducing a second optimization process for the numerical minimization of the Worst-case Convergence Rate of the SLiSe rational filters. The byproduct of such minimization is the elimination of the dependence of the filter from the weight functions used in the non-linear least squares functional. The new WiSe filters outperform GAussLEGENDRE and ZOLOTAREV filters both in terms of execution time, number of subspace iterations, and FLOPs count necessary to reach convergence by the eigensolver.

Increasing the performance of the optimization of rational filters and eliminating their dependence from a number of adjustable parameters has an additional indirect and important impact on the eigensolver using the rational filters as spectral projectors. This class of solvers lend themselves to multiple levels of parallelism: At the highest level each interval $[a, b]$ can be split in subintervals $\left[a_{j}, b_{j}\right]$, each of which constitutes a trivially separate eigenproblem; at a mid-level the spectral solver requires the solution of a linear system for each pole $z_{i}$; at the lowest level each linear system has to be solved for multiple RHS. While such a general scheme makes this class of eigensolver attractive, it complicates substantially the problem of balancing the computational load. One of the main contributor to the uncertainty of a well-balanced computation is the ability of the spectral filter in determining the number of subspace iterations needed to converge the full subspace corresponding to each $\left[a_{j}, b_{j}\right]$.

Our WiSe filters overcome this uncertainty by: 1) decoupling the rate of convergence from the size of the active search subspace, 2) drastically reducing the dependence of the number of poles which can be safely set to a standard value (e.g. $m=4$ in FEAST). The net result is that the spectral filter has the same effectiveness for any sub-interval selected: For a given linear system solver the number of iterations required to reach convergence is minimized and independent from the eigenvalues distribution. Load balancing is then achieved by choosing sub-intervals with approximately the same eigenvalue count. Since obtaining a good estimate for the eigenvalue count and the eigenvalue distribution is a solved problem [5, 22], the result presented in this paper eliminates the influence of the spectral filter on load balancing for all practical purposes. The remaining challenge is balancing the load when solving for distinct linear system with multiple RHS. This is the focus of further ongoing work.

Acknowledgements. We thank Jan Winkelmann for having provided support to the first author in developing the bulk of the work that contributed to this paper and Sebastian Achilles for useful discussions.

\section{REFERENCES}

[1] M. V. BARel, Designing Rational Filter Functions for Solving Eigenvalue Problems by Contour Integration, Linear Algebra Appl., 502 (2016), pp. 346 - 365.

[2] B. Beckermann And A. Townsend, On the singular values of matrices with displacement structure, SIAM Journal on Matrix Analysis and Applications, 38 (2017), pp. 1227-1248, https://doi.org/10.1137/16M1096426.

[3] R. Byrd, P. Lu, J. Nocedal, and C. Zhu, A limited memory algorithm for bound constrained optimization, SIAM Journal on Scientific Computing, 16 (1995), pp. 1190-1208, https: //doi.org/10.1137/0916069.

[4] T. A. Davis And Y. Hu, The University of Florida Sparse Matrix Collection, ACM Transactions on Mathematical Software, 38 (2011), pp. 1:1-1:25, https://doi.org/10.1145/2049662. 2049663.

[5] E. Di NApoli, E. Polizzi, ANd Y. SAAD, Efficient estimation of eigenvalue counts in an interval, Numerical Linear Algebra with Applications, 23 (2016), pp. 674-692, https: //doi.org/10.1002/nla.2048. 
[6] E. P. ET AL., FEAST eigenvalue solver, version 3.0, http://www.feast-solver.org/.

[7] R. Feldt, Black-box optimization for Julia, https://github.com/robertfeldt/BlackBoxOptim.jl. version 0.3.0.

[8] Y. Futamura and T. SAKurai, $z$-Pares: Parallel eigenvalue solver, http://zpares.cs.tsukuba. ac.jp/.

[9] B. Gavin And E. Polizzi, Krylov eigenvalue strategy using the FEAST algorithm with inexact system solves, Numerical Linear Algebra with Applications, (2018), p. e2188, https://doi.org/10.1002/nla.2188. To be included.

[10] D. Göddeke, Fast and Accurate Finite-Element Multigrid Solvers for PDE Simulations on GPU Clusters, PhD thesis, Technische Universität Dortmund, 2011.

[11] J. Gopalakrishnan, L. Grubišić, and J. Ovall, Filtered subspace iteration for selfadjoint operators, ArXiv e-prints, (2017), https://arxiv.org/abs/1709.06694.

[12] S. Güttel, E. Polizzi, P. TAng, ANd G. Viaud, Zolotarev quadrature rules and load balancing for the FEAST eigensolver, SIAM Journal on Scientific Computing, 37 (2015), pp. A2100-A2122, https://doi.org/10.1137/140980090.

[13] T. Ikegami and T. Sakurai, Contour Integral Eigensolver for non-Hermitian Systems: A Rayleigh-Ritz-type Approach, Taiwanese J. Math., 14 (2010), pp. pp-825.

[14] T. Inegami, T. Sakurai, and U. Nagashima, A Filter Diagonalization for Generalized Eigenvalue Problems Based on the Skaurai-Sugiuara Projection Method, J. Comput. Appl. Math., 233 (2010), pp. 1927 - 1936.

[15] A. Imakura, L. Du, and T. Sakurai, Error bounds of Rayleigh-Ritz type contour integralbased eigensolver for solving generalized eigenvalue problems, Numerical Algorithms, 71 (2016), pp. 103-120, https://doi.org/10.1007/s11075-015-9987-4.

[16] S. G. Johnson, The NLopt nonlinear-optimization package, http://ab-initio.mit.edu/nlopt.

[17] C. Kelley, Detection and remediation of stagnation in the Nelder-Mead algorithm using a sufficient decrease condition, SIAM Journal on Optimization, 10 (1999), pp. 43-55, https://doi.org/10.1137/S1052623497315203.

[18] L. Krämer, E. Di Napoli, M. Galgon, B. Lang, and P. Bientinesi, Dissecting the FEAST algorithm for generalized eigenproblems, Journal of Computational and Applied Mathematics, 244 (2013), https://doi.org/10.1016/j.cam.2012.11.014.

[19] L. Krämer AND B. LANG, Concergence of integration-based methods for the solution of standard and generalized hermitian eigenvalue problems, Electronic Transactions on Numerical Analysis, 48 (2018), p. 183-201, https://doi.org/10.1553/etna_vol48s183.

[20] J. Lagarias, J. Reeds, M. Wright, and P. Wright, Convergence properties of the nelder-mead simplex method in low dimensions, SIAM Journal on Optimization, 9 (1998), pp. 112-147, https://doi.org/10.1137/S1052623496303470.

[21] R. Li, Y. XI, L. ERLandson, And Y. SAAD, The Eigenvalues Slicing Library (EVSL): Algorithms, Implementation, and Software, ArXiv e-prints, (2018), https://arxiv.org/abs/ 1802.05215 .

[22] L. Lin, Y. SAAD, And C. YAng, Approximating spetral densities of large matrices, SIAM Review, 58 (2016), pp. 34-65, https://doi.org/10.1137/130934283.

[23] K. McKinnon, Convergence of the nelder-mead simplex method to a nonstationary point, SIAM Journal on Optimization, 9 (1998), pp. 148-158, https://doi.org/10.1137/ S1052623496303482.

[24] J. L. Morales And J. Nocedal, Remark on Algorithm 778: L-BFGS-B: Fortran subroutines for large-scale bound constrained optimization, ACM Transactions on Mathematical Software, 38 (2011), pp. 7:1-7:4, https://doi.org/10.1145/2049662.2049669.

[25] H. Murakami, An Experiment of the Filter Diagonalization Method for the Banded Generalized Symmetric-Definite Eigenproblem, Tech. Report 59(2007-HPC-110), Tokyo Metropolitan University, 2007.

[26] H. Murakami, A Filter Diagonalization Method by the Linear Combination of Resolvents, IPSJ Trans. ACS, 49 (2008), pp. 66-87.

[27] H. Murakami, The Filter Diagonalization Method for the Unsymmetric Matrix Eigenproblem, Tech. Report 43(2008-HPC-115), Tokyo Metropolitan University, 2008.

[28] H. Murakami, Experiments of Filter Diagonalization Method for Real Symmetric Definite Generalized Eigenproblems by the use of Elliptic Filters, Tech. Report 2010-HPC-125, Tokyo Metropolitan University, jun 2010.

[29] H. Murakami, Optimization of Bandpass Filters for Eigensolver, Tech. Report 2010-HPC-124, Tokyo Metropolitan University, 2010.

[30] J. Nocedal and S. Wright, Numerical Optimization, Springer-Verlag, 2 ed., 7 2006, https: //doi.org/10.1007/978-0-387-40065-5.

[31] E. Polizzi, Density-matrix-based algorithm for solving eigenvalue problems, Physical Review 
B, 79 (2009), pp. 115112-115117, https://doi.org/10.1103/PhysRevB.79.115112.

[32] T. Sakurai And H. Sugiura, A projection method for generalized eigenvalue problems using numerical integration, Journal of Computational and Applied Mathematics, 159 (2003), pp. 119 - 128, https://doi.org/10.1016/S0377-0427(03)00565-X.

[33] T. Sakurai and H. Tadano, CIRR: a Rayleigh-Ritz type method with contour integral for generalized eigenvalue problems, Hokkaido Mathematical Journal, 36 (2007), pp. 745-757, https://doi.org/10.14492/hokmj/1272848031.

[34] L. Sorber, M. V. Barel, and L. D. Lathauwer, Unconstrained optimization of real functions in complex variables, SIAM Journal on Optimization, 22 (2012), pp. 879-898, https://doi.org/10.1137/110832124.

[35] P. TAng and E. Polizzi, FEAST as a subspace iteration eigensolver accelerated by approximate spectral projection, SIAM Journal on Matrix Analysis and Applications, 35 (2014), pp. 354390, https://doi.org/10.1137/13090866X.

[36] J. Winkelmann and E. Di Napoli, Non-Linear Least-Squares Optimization of Rational Filters for the Solution of Interior Eigenvalue Problems, ArXiv e-prints, (2017), https: //arxiv.org/abs/1704.03255.

[37] Y. XI And Y. SAAD, Computing Partial Spectra with Least-Squares Rational Filters, SIAM J. Sci. Comput., 38 (2016), pp. A3020-A3045.

[38] C. Zhu, R. Byrd, P. Lu, and J. Nocedal, Algorithm 778: L-BFGS-B: Fortran subroutines for large-scale bound-constrained optimization, ACM Transactions on Mathematical Software, 23 (1997), pp. 550-560, https://doi.org/10.1145/279232.279236. 